\title{
The modelling of carious lesion progress
}

\author{
$\underline{\text { Tadeusz Kosztołowicz }}^{\text {a }}$, Katarzyna D. Lewandowska $^{\text {b }}$ \\ ${ }^{a}$ Institute of Physics, Jan Kochanowski University, \\ ul. Świętokrzyska 15, 25-406 Kielce, Poland \\ ${ }^{\mathrm{b}}$ Department of Radiological Informatics and Statistics, Medical University of Gdańsk, \\ ul. Tuwima 15, 80-210 Gdańsk, Poland \\ Email: tadeusz.kosztolowicz@ujk.edu.pl
}

\begin{abstract}
A carious lesion in the tooth enamel is a result of a chemical reaction between mobile organic acids and static hydroxyapatite. Hydroxyapatite is the main component of human tooth enamel, whereas, organic acids are produced in dental plaque by oral microorganisms which metabolize simple sugars from the diet. The organic acids can be transported into the enamel in a dissociated or undissociated form, which depends on the $\mathrm{pH}$ of the dental plaque. The transport of ions is diffusive and is motivated by the small diameter of the hydrogen ions compared to the size of the enamel tubules, whereas, when large acid molecules move into the enamel, subdiffusion occurs.
\end{abstract}

Diffusion is a natural process involving the spontaneous spreading of a substance. Diffusion is characterized by the time dependence of the mean-square displacement of a random walker $\left\langle(\Delta x)^{2}(t)\right\rangle=D_{\alpha} t^{\alpha} / \Gamma(1+\alpha)$, where $\alpha$ is a diffusion parameter, $D_{\alpha}$ is a diffusion coefficient measured in the units of $\mathrm{m}^{2} / \mathrm{s}^{\alpha}$ and $\Gamma(x)$ denotes the Gamma function. For $0<\alpha<1$ we are dealing with subdiffusion; for $\alpha=1$, we have a situation of normal diffusion, and for $\alpha>1$, we encounter superdiffusion. Subdiffusion occurs in media in which the movement of the random walker is strongly hindered due to the complex structure of a medium and subdiffusion occurs — among other things — in porous media or gels.

Equations describing the subdiffusive transport of acid molecules and their reaction with static hydroxyapatite are nonlinear partial differential equations with the Riemann-Liouville fractional time derivative

$$
\begin{aligned}
& \frac{\partial C_{A}(x, t)}{\partial t}=D_{\alpha} \frac{\partial^{1-\alpha}}{\partial t^{1-\alpha}} \frac{\partial^{2} C_{A}(x, t)}{\partial x^{2}}-R_{\alpha}(x, t) \\
& \frac{\partial C_{B}(x, t)}{\partial t}=-R_{\alpha}(x, t)
\end{aligned}
$$

where $C_{A}$ denotes the concentration of a mobile substance $A, C_{B}$-the static substance $B$ and the reaction term takes the form

$$
R_{\alpha}(x, t)=\frac{\partial^{1-\alpha}}{\partial t^{1-\alpha}} k C_{A}(x, t) C_{B}(x, t)
$$

and $k$ is the reaction rate constant. For $\alpha=1$ we obtain equations describing a normal diffusion-reaction system. As far as we know, the general solutions, i.e. for arbitrary parameter values, to (sub)diffusion-reaction equations have not been found yet. Thus, in order to simplify the calculations, various approximations, such as the quasistationary approximation, the scaling method, or the perturbation method, are used. Employing these methods, characteristic functions of the system can be derived which include, among others things, the time evolution of the reaction front which can be identified with a lesion depth.

Using the perturbation method we find approximate solutions to the normal diffusion-reaction equations which are quite satisfactory in comparison with experimental data. We will also find that the time evolution of the lesion depth in the cases of acid transport, normal diffusion and subdiffusion, is a power function of time $x_{f} \sim t^{\alpha / 2}$. This result is also in accordance with the experimental data.

Keywords: carious lesion, normal diffusion-reaction process, subdiffusion-reaction process, perturbation method, scaling method 


\section{INTRODUCTION}

Diffusion is the natural process of the spontaneous spreading of a substance. Diffusion is characterized by the time dependence of the mean-square displacement of a random walker which reads $\left\langle(\Delta x)^{2}(t)\right\rangle=$ $D_{\alpha} t^{\alpha} / \Gamma(1+\alpha)$, where $\alpha$ is a diffusion parameter, $D_{\alpha}$ is a diffusion coefficient measured in the units of $\mathrm{m}^{2} / \mathrm{s}^{\alpha}$ and $\Gamma(x)$ denotes the Gamma function, Metzler and Klafter (2000). For $0<\alpha<1$ we are dealing with subdiffusion; for $\alpha=1$, we have a situation of normal diffusion, and for $\alpha>1$, we encounter superdiffusion. Subdiffusion occurs in media in which the movement of the random walker is strongly hindered due to the complex structure of a medium and such subdiffusion occurs, among other things, in porous media or gels, Kosztołowicz et al. (2005). Normal diffusion as well as anomalous diffusion are both described by a partial differential equation (in the latter case with a fractional time derivative).

Now we present the description of the process of caries which is based on the series of papers cited in Kosztołowicz and Lewandowska (2006); Lewandowska and Kosztołowicz (2012). The formation of carious lesions in the enamel starts when the concentration of organic acids in the dental plaque reaches a sufficient value and the $\mathrm{pH}$ of the dental plaque lowers to below an appropriate point. Then organic acids diffuse into the enamel and react with the mineral to form soluble calcium ions and phosphate ions (or complexes). It is commonly accepted that the products of the reaction diffuse out of the enamel. Organic acids (e.g. acetic or lactic) are produced in the dental plaque by oral microorganisms that metabolize the simple sugars coming from the diet. In the first stage of carious lesion formation an apparently intact surface layer can be created. This is the layer where the loss of mineral is small in comparison to the content of the mineral in the sound enamel. First of all, this loss reveals the formation of tiny holes. Two possible mechanisms responsible for creating the surface layer have been proposed. Firstly, the appearance of protective agents prevents acids from dissolving the enamel. Second, the surface layer exists as a result of the combination of dissolution and reprecipitation processes. The thickness of this layer reaches maximum value and remains unchanged later on. Dissolution of subsurface mineral (situated below the apparently intact surface layer) occurs in the second stage of the formation of the carious lesion according to the chemical formula $A+B \rightarrow \emptyset$ (inert) where $A$ denotes a mobile substance and $B$ - a static substance. However, before the dissolution of the enamel, acids have to reach the enamels interior. The enamel is built out of crystal of hydroxyapatite (HA). These crystals are organized in larger forms called prisms. The intercrystalline and interprismatic spaces of the enamel are filled with water. Thus, we can say that the enamel is similar to a porous medium. The acids diffuse from dental plaque through pores of enamel below the surface layer. The acids can diffuse in dissociated or undissociated forms that depend on the $\mathrm{pH}$ of the dental plaque. The transport of ions inside the enamel is diffusive, a process which is motivated by the small diameter of hydrogen ions compared to the size of the enamel tubules, whereas when large acid molecules move inside the enamel, subdiffusion occurs.

To date, many scientists have focused their attention on the theoretical description of the process of caries. However, the problem is not simply in regard to the complexly of this process. Namely, a lot of factors can influence the carious lesion process such as age, environment, hygiene, the diet, etc., Zero (1999). For this reason, assumptions which can oversimplify the problem are usually made. Some research has considered only one of the equations from among (1) and (2). Additionally, the reaction term $R_{\alpha}$ was taken in an oversimplified form, in which only one reactant is taken into account. For example, in the paper of Wu et al. (1976), Eq. (1) alone was studied, with the reaction term in the form $R=k\left(C_{\mathrm{s}}-C\right)$, where $C_{\mathrm{s}}$ is a constant related to the solubility of the solute. In Maksimovskii et al. (1990) the reaction term was chosen in the form $R(x, t)=$ $k(x, t) A(x, t)$. A model based on Eq. (2) alone was studied in Gray (1966) with $R(x, t)=k C^{\mathrm{n}}$, and in BolletQuivogne et al. (2005) with $R(x, t)=k\left(C_{\mathrm{s}}-C\right)$. Equations (1) and (2) together were considered in Shi and Erickson (2001) with $R(x, t)=k A$. We should add here that the models based only on the normal diffusion equation without chemical reactions included through the 'effective' diffusion coefficient were also considered, Chu et al. (1989). Very special theoretical diffusive models were used to describe some caries characteristics, such as the time evolution of the caries depth, Christoffersen and Arends (1982), and the concentration profiles of hydrogen ions, Maksimovskii et al. (1990) inside the enamel in a stationary state. As far as we know, a model which takes into account the time evolutions of both the concentration of a mobile substance and the concentration of a static substance have been proposed for the first time in Lewandowska and Kosztołowicz (2012).

In this paper, our purpose is to present a theoretical model of carious lesion process which is more realistic, general and which satisfactorily approximates the experimental data. Unfortunately, the experimental data concerning different aspects of carious lesions is very infrequent, especially in that it is difficult to find the enamel concentration profiles. In our model we use Eq. (1) which describes the transport of mobile sub- 
stance $A$ and chemical reaction with static substance $B$, Eq. (2) which only describes the reaction of static substance $B$ with the mobile substance $A$ and the reaction term (3), which describes chemical reactions between substances $A$ and $B$. In particular, we propose two theoretical models of the carious lesion process. One of them describes diffusive transport of a dissociated acid and its chemical reaction with the mineral, the second one-the subdiffusive transport of an undissociated acid and its chemical reaction with the mineral. (Sub)diffusion-reaction equations, which we use in modelling the transport of hydrogen ions or acid molecules and their reactions with static hydroxyapatite are nonlinear partial differential equations (in the case of subdiffusion with a fractional time derivative). As far as we know, the general solutions, i.e. for arbitrary parameter values, to (sub)diffusion-reaction equations have not yet been found. Thus, in order to simplify calculations, various approximations, such as the quasistationary approximation, the scaling method or the perturbation method, are used.

Below, we consider the normal diffusion-reaction and the subdiffusion-reaction cases separately, with regard for different calculation techniques. In the case of the diffusive transport of dissociated acid we use the perturbation method in order to find the concentration profiles of the enamel mineral and the time evolution of the lesion depth. In the case of the subdiffusive transport of acid in an undissociated form we find the time evolution of the lesion depth. In both cases of acid transport, normal diffusion and subdiffusion, is a power function of time $x_{f} \sim t^{\alpha / 2}$.

\section{Model}

The system under study is three-dimensional, but it is assumed to be homogeneous in the plane perpendicular to the $x$-axis, which is normal for the surface of a tooth. Thus, the system can be treated as one-dimensional. The scheme of the system under consideration is presented in Fig. 1 which is based on the qualitative description and the plots of experimental concentration profiles given in the papers cited in Kosztołowicz and Lewandowska (2006); Lewandowska and Kosztołowicz (2012). In Fig. 1 we can discern two regions denoted

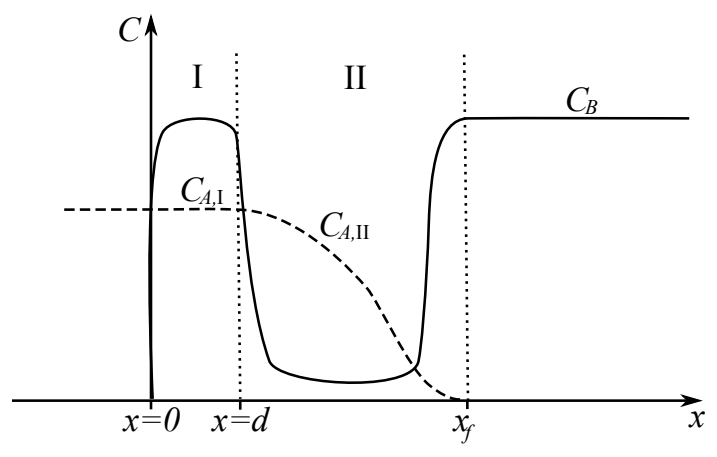

Figure 1. The schematic view of the system; the solid line represents the concentration of the static mineral, and the dashed one- the concentration of the acid. A more detailed description is in the text.

as I and II, respectively. These regions are built of static substance $B$ and —at the initial moment-are free of any mobile substance. Region I corresponds with the surface layer of the enamel (which, as already mentioned, is not created in all caries). Region II is called a subsurface layer. Within this layer, chemical reactions occur and the amount of substance $B$ decreases as a result of the demineralization process. The mobile substance is denoted by $A, \mathrm{I}$ in region I and by $A, \mathrm{II}$ in region II. Substance $A$ only moves (sub)diffusively through region I, whereas, in region II (sub)diffusion and chemical reactions occur.

As already mentioned, substances $A$ and $B$ are separated at the initial moment. Thus, the initial conditions $\operatorname{read} C_{A, \mathrm{I}}(x, 0)=0$ for $x \in(0, d), C_{A, \mathrm{II}}(x, 0)=0$ for $x \in(d, \infty)$ and $C_{B}(x, 0)=C_{0 B}$ for $x \in(0, \infty)$. We assume that the reservoir of substance $A$ occurs for $x<0$. Therefore, the concentration of substance $A$ is constant at $x=0$. Moreover, the thickness of regions I and II composes a small part of the enamel and it is quite obvious that concentrations and fluxes of substance $A$ are continuous at the border between regions I and II. So, we take the following boundary conditions $C_{A}(0, t)=C_{0 A}, C_{A, \mathrm{I}}(d, t)=C_{A, \mathrm{II}}(d, t)$, $\partial C_{A, \mathrm{I}}(x, t) /\left.\partial x\right|_{x=d}=\partial C_{A, \mathrm{II}}(x, t) /\left.\partial x\right|_{x=d}$ and $C_{A, \mathrm{II}}(\infty, t)=0$. 
Equations which describe the subdiffusive transport of acid molecules and their reaction with static hydroxyapatite are nonlinear partial differential equations with a fractional derivative

$$
\begin{aligned}
\frac{\partial C_{A, \mathrm{I}}(x, t)}{\partial t} & =D_{\alpha} \frac{\partial^{1-\alpha}}{\partial t^{1-\alpha}} \frac{\partial^{2} C_{A, \mathrm{I}}(x, t)}{\partial x^{2}}, \quad x \in(0, d) \\
\frac{\partial C_{A, \mathrm{II}}(x, t)}{\partial t} & =D_{\alpha} \frac{\partial^{1-\alpha}}{\partial t^{1-\alpha}} \frac{\partial^{2} C_{A, \mathrm{II}}(x, t)}{\partial x^{2}}-R_{\alpha}(x, t), \quad x \in(d, \infty), \\
\frac{\partial C_{B}(x, t)}{\partial t} & =-R_{\alpha}(x, t), \quad x \in(d, \infty)
\end{aligned}
$$

where $d^{\alpha} f(t) / d t^{\alpha}$ denotes the Riemann-Liouville fractional time derivative which is defined as for $\alpha>0$ $d^{\alpha} f(t) / d t^{\alpha}=(1 / \Gamma(n-\alpha))\left(d^{n} / d t^{n}\right) \int_{0}^{t} d t^{\prime} f\left(t^{\prime}\right) /\left(t-t^{\prime}\right)^{1+\alpha-n}$, where $n$ is the smallest natural number which fulfils the equation $n-\alpha>0 ; D_{\alpha}$ is a subdiffusion coefficient and the reaction term takes the form $R_{\alpha}(x, t)=\partial^{1-\alpha} k C_{A, \mathrm{II}}(x, t) C_{B}(x, t) / \partial t^{1-\alpha}$, and $k$ is the reaction rate constant. For $\alpha=1$ we obtain equations describing a normal diffusion-reaction system.

Below, we study the two detailed models (normal diffusion and subdiffusion) which satisfactorily approximate the experimental data, Kosztołowicz and Lewandowska (2006); Lewandowska and Kosztołowicz (2012). In the first model utilizing normal diffusion-reaction equations (Eqs. (4)-(6) for $\alpha=1$ ), we use the perturbation method in order to find the enamel concentration profiles. The obtained theoretical profiles fit the experimental concentrations well, thus we present this model in sec. 3. In the second model we employ subdiffusionreaction equations (Eqs. (4)-(6) for $0<\alpha<1$ ) and we determine the time evolution of the caries depth by means of the scaling method. The obtained relation also fits the experimental data well. We present this model in sec. 4.

\section{NORMAL DIFFUSION MODEL}

In this section we will study a system in which substance $A$ moves diffusively within the regions I and II. This corresponds to a situation in which organic acid occurs in a dissociated form. Equations describing the system are (4)-(6) for $\alpha=1$. As already mentioned, these equations are nonlinear partial differential equations and their general solutions remain unknown (except in very special cases). Therefore, we use the perturbation method in order to solve the above equations, Taitelbaum et al. (1996).

The perturbation method is usually used when equations are in the form $\partial f / \partial x=\mathcal{F}(f)+\varepsilon \mathcal{G}(f)$, where $\varepsilon$ is a dimensionless small parameter and it is assumed that the solution to the equation $\partial f_{0} / \partial x=\mathcal{F}\left(f_{0}\right)$ is known. Then, the solution to equation $\partial f / \partial x=\mathcal{F}(f)+\varepsilon \mathcal{G}(f)$ is a power series with respect to the parameter $\varepsilon$ and reads $f=\sum_{n=0}^{\infty} \varepsilon^{n} f_{n}$.

We have solved Eqs. (4)-(6) by means of the perturbation method. The details of calculation are presented in Lewandowska and Kosztołowicz (2012). Below, we only present the concentration profiles of a mobile substance $A, \mathrm{I}$ in region I and $A, \mathrm{II}$ in region II, and a static substance $B$ calculated as a sum of the zeroth order and first order concentrations.

$$
\begin{aligned}
C_{A, \mathrm{I}}(x, t)= & C_{0 A} \operatorname{erfc}\left(\frac{x}{2 \sqrt{D_{1} t}}\right)+k C_{0 B}\left[\frac{1}{4}\left(t+\frac{(2 d+x)^{2}}{2 D_{1}}\right) \operatorname{erfc}\left(\frac{2 d+x}{2 \sqrt{D_{1} t}}\right)-\frac{(2 d+x) \sqrt{t}}{\sqrt{\pi D_{1}}} e^{-\frac{(2 d+x)^{2}}{4 D_{1} t}}\right. \\
& -\left(t+\frac{(2 d-x)^{2}}{2 D_{1}}\right) \operatorname{erfc}\left(\frac{2 d-x}{2 \sqrt{D_{1} t}}\right)+\frac{(2 d-x) \sqrt{t}}{\sqrt{\pi D_{1}}} e^{\left.-\frac{(2 d-x)^{2}}{4 D_{1} t}\right]} \\
C_{A, \mathrm{II}}(x, t)= & C_{0 A} \operatorname{erfc}\left(\frac{x}{2 \sqrt{D_{1} t}}\right)+k C_{0 B}\left[\frac{1}{4}\left(t+\frac{(2 d+x)^{2}}{2 D_{1}}\right) \operatorname{erfc}\left(\frac{2 d+x}{2 \sqrt{D_{1} t}}\right)-\frac{(2 d+x) \sqrt{t}}{\sqrt{\pi D_{1}}} e^{-\frac{(2 d+x)^{2}}{4 D_{1} t}}\right. \\
& \left.-\left(t+\frac{(4 d-3 x) x}{2 D_{1}}\right) \operatorname{erfc}\left(\frac{x}{2 \sqrt{D_{1} t}}\right)+\frac{(4 d-3 x) \sqrt{t}}{\sqrt{\pi D_{1}}} e^{-\frac{(2 d-x)^{2}}{4 D_{1} t}}\right] \\
C_{B}(x, t)= & C_{0 B}-C_{0 A} C_{0 B} k\left[\left(t+\frac{x^{2}}{2 D_{1}}\right) \operatorname{erfc}\left(\frac{x}{2 \sqrt{D_{1} t}}\right)-x \sqrt{\frac{t}{\pi D_{1}}} e^{-x^{2} / 4 D_{1} t}\right]
\end{aligned}
$$

where $\operatorname{erfc}(u)=(2 / \sqrt{\pi}) \int_{u}^{\infty} \exp \left(-\eta^{2}\right) d \eta$ is the complementary error function. These solutions are in quantitatively and qualitatively good agreement with the experimental data, see Figs. 2 and 3 in Lewandowska and Kosztołowicz (2012). Thus, the perturbation method including in its calculation the zeroth order and the first order alone presents well enough in modelling the carious lesion process. 


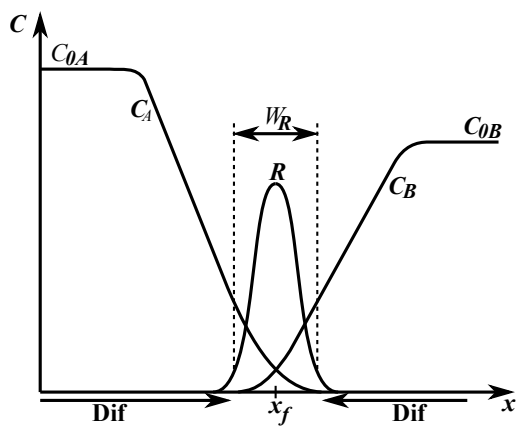

Figure 2. The schematic view of the subdiffusion-reaction system; $R$ denotes the reaction region, $W_{R}-$ the reaction region width and 'Dif' stands for the diffusion region.

\section{Subdiffusion MOdel}

Below we will study a system in which the surface layer does not occur, thus, $x=d=0$ and the mobile substance $A$ moves subdiffusively within region II. This corresponds to a situation in which an organic acid occurs in an undissociated form. Equations describing such a system are (5) and (6). The initial conditions and the boundary conditions read

$$
C_{A}(x, 0)=0, \quad C_{B}(x, 0)=C_{0 B}, \quad \text { and } \quad C_{A}(0, t)=C_{0 A}, \quad C_{A}(\infty, t)=0 .
$$

It should be mentioned here that when the subdiffusion-reaction process starts, characteristic regions and points appear in the system (see Fig. 2), Kosztołowicz and Lewandowska (2008) and references cited therein. One of these characteristic regions is reaction region $R$ which is a part of the system in which the reaction is the most intense. Outside this region we can assume $R_{\alpha}(x, t) \approx 0$. Thus, in this part of the system, which is called the diffusion region 'Dif', only a subdiffusive transport occurs. The most characteristic point inside the reaction region is reaction front $x_{f}$, which can be defined as a point at which the reaction term $R_{\alpha}$ reaches its maximum. The reaction front can be identified with the depth of the carious lesion.

As already mentioned, subdiffusion-reaction equations are nonlinear partial differential equations with fractional time derivatives. Solutions to these equations have not yet been found, that is, with the exception of a few very special cases. For the subdiffusion-reaction system with a static substance and a mobile substance we have calculated the concentration profiles using the scaling method, Kosztołowicz et al. (2013). For the case of both mobile substances we have found the concentration profiles in the diffusion region and the exact formula for the time evolution of the reaction front by means of the quasistationary method, Kosztołowicz and Lewandowska (2008). However, we cannot obtain the solutions to subdiffusion-reaction equations in the case of one static and one mobile substance, by merely setting one of the subdiffusion coefficients equal to zero, as this gives a system without any chemical reactions. Thus, in order to find solutions to Eqs. (5) and (6) we use the scaling method, Bazant and Stone (2000).

The idea of the scaling method is as follows. Let us assume that the scale relations $x^{\prime}=a^{\delta} x, t^{\prime}=a^{\beta} t$ and $C\left(x^{\prime}, t^{\prime}\right)=a^{\gamma} C(x, t)$, where $a$ is a positive constant and $\delta, \beta$ and $\gamma$ are unknown parameters, are valid. If the equations describing the system, the boundary conditions and the initial conditions do not change their forms under these transformations, then the solutions can be expressed by the following function $C(x, t)=$ $t^{\gamma / \beta} \Phi(\eta)$, where $\eta$ is an invariant combination of the variables $x$ and $t$ in the form such as, for example, $\eta_{1}=\left(x+2 \nu t^{\sigma}\right) / t^{\delta / \beta}$ or $\eta_{2}=\left(x+2 \nu t^{\sigma}\right) / 2 t^{\delta / \beta}$, where $\nu$ is a constant and $\sigma$-an unknown parameter. Next, after substituting $C(x, t)=t^{\gamma / \beta} \Phi(\eta)$ into the equations under consideration, the values of parameters $\delta, \beta, \gamma$ and $\sigma$ can be determined by comparison between the appropriate exponents of dominating terms.

In the paper Kosztołowicz and Lewandowska (2006), in which we have studied the system describing by Eqs. (5), (6) and (10) all calculation details can be found. We should mention here, that functions present in different parts of the subdiffusion-reaction system, all scale according to different rules. This has been confirmed by the numerical calculations. Therefore, in the subdiffusion-reaction system, with one static substance, e.g. $B$, the concentrations within the reaction region scales according to the formula $C_{A}(x, t)=t^{-\alpha / 2} \tilde{C}\left(\eta_{1}\right)$ and $C_{B}(x, t)=\tilde{C}\left(\eta_{1}\right)$ whereas the concentration within the diffusion region is $C_{A}(x, t)=\tilde{C}\left(\eta_{2}\right)$. Moreover, all 


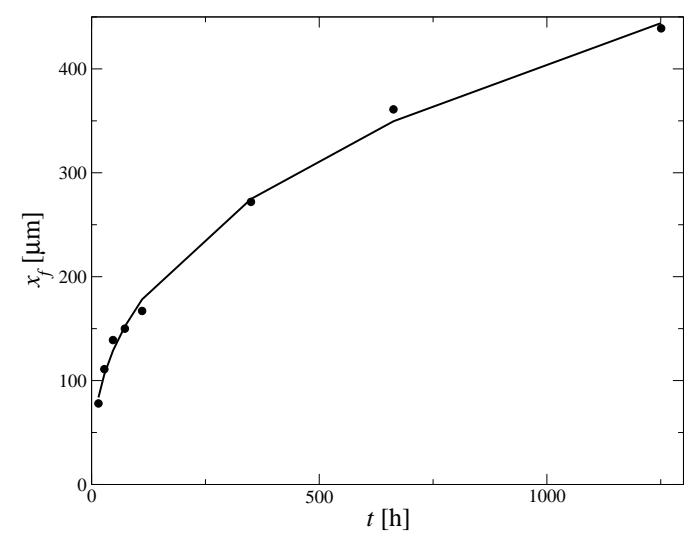

Figure 3. Carious lesion depth versus time. The circles represent the experimental data whereas the solid line is the plot of the power function (12).

calculations are complicated and greatly tiresome because of the presence of fractional derivatives. The main result obtained in that paper is the derivation of the following relation

$$
x_{f} \sim t^{\alpha / 2} .
$$

Thus, the time evolution of the reaction front in the system with one static substance and one mobile substance is the power function with an exponent depending on the subdiffusion parameter $\alpha$ alone. Moreover, the time evolution of the reaction front (11) does not depend on the form of the reaction term, thus it appears to be a general property of the subdiffusion-reaction process. In the following, we identify the reaction front with the caries depth.

In Fig. 3 we present the time evolution of the caries depth which was measured experimentally (circles) and which we have taken from Featherstone et al. (1979), and the power function (solid line) calculated form the formula

$$
x_{f}=30.11 \times t^{0.38}
$$

which is the best fit to the experimental data obtained by means of the least squares method. Comparing the functions (11) and (12) we find $\alpha=0.76$, which means that subdiffusion occurs in the enamel in the case under study.

\section{CONCLUSIONS}

The theoretical model based on Eqs. (4)-(6) has a general character. The models, which have been presented here, are its particular cases, and have been adopted in order to approximate the experimental data. As already mentioned, the carious lesion progress has an individual character and depends on many factors. In our opinion, the theoretical models presented here can be easily adjusted to these individual situations by, for example, the modification of the reaction term, the subdiffusion parameter, the (sub)diffusion coefficient, etc. or by making parameters dependent on time. In this way, many factors, such as more complicated chemical reactions, the attendance of inhibitors and so on, can be included in the model. Moreover, in the cases of equations which can be too complicated for analytical treatment, numerical methods can be used. The details of this method and numerical solutions to subdiffusion-reaction equations are presented in Lewandowska and Kosztołowicz (2007); Kosztołowicz and Lewandowska (2008).

We have shown that the perturbation method can be useful in the modelling of carious lesion progress. A comparison of the analytic solutions with the experimental data allow us to determine the value of the acid ions diffusion coefficient, namely $D_{1}=2.2 \times 10^{-10} \mathrm{~cm}^{3} / \mathrm{s}$, Lewandowska and Kosztołowicz (2012). We should note that the obtained value of the diffusion coefficient takes 'typical' values. For example it was reported in van Dijk J.W. et al. (1983) that $D \propto 10^{-8}-10^{-13} \mathrm{~cm}^{2} / \mathrm{s}$ for several substances diffusing in the tooth enamel.

We have shown that the carious lesion depth evolves over time as $x_{f} \sim t^{\alpha / 2}$ with $\alpha \leq 1$. This relation can be used in order to identify a kind of transport process occurring in the enamel. We fit formula (11) with 
the experimental data presenting the caries depth, which we discovered in the literature. We have found the carious lesion depth evolving over time $x_{f} \sim t^{\alpha / 2}$, where $0<\alpha<1$ (this means the subdiffusive transport of acids), as well as the carious lesion depth evolving over time $x_{f} \sim t^{1 / 2}$ (this means the diffusive transport of acids). Let us also remember here that acids can be transported diffusively (dissociated acids) or subdiffusively (undissociated acids). The form of acids, dissociated or undissociated, depends on the $\mathrm{pH}$ of the dental plaque.

\section{REFERENCES}

Bazant, M. and H. Stone (2000). Asymptotic of reaction-diffusion fronts with one static and one diffusing reactant. Physica D 147, 95-121.

Bollet-Quivogne, F., P. Anderson, S. Dowker, and J. Elliott (2005). Scanning microradiographic study on the influence of diffusion in the external liqiud on the rate of demineralization in hydroxyapatite aggregates. Eur. J. Oral Sci. 113, 53-59.

Christoffersen, J. and J. Arends (1982). Progress of artificial carious lesions in enamel. Caries Res. 16(6), 433-439.

Chu, J., J. Fox, and W. Higuchi (1989). Quantitative study of fluoride transport during subserface dissolution of dental enamel. J. Dent. Res. 68(1), 32-41.

Featherstone, J., J. Duncan, and T. Cutress (1979). A mechanism for dental caries based on chemical processes and diffusion phenomena during in-vitro caries simulation on human tooth enamel. Archs. Oral Biol. 24, $101-112$.

Gray, J. (1966). Kinetics of enamel dissolution during formation of incipient caries-like lesions. Arch. Oral Biol. 11, 397-421.

Kosztołowicz, T., K. Dworecki, and S. Mrówczyński (2005). How to measure subdiffusion parameters. Phys. Rev. Lett. 94, 170602.

Kosztołowicz, T. and K. Lewandowska (2006). Time evolution of the reaction front in the system with one static and one subdiffusive reactant. Acta Phys. Pol. B 37(5), 1571-1578.

Kosztołowicz, T. and K. Lewandowska (2008). Time evolution of the reaction front in a subdiffusive system. Phys. Rev. E 78, 066103.

Kosztołowicz, T., M. Piwnik, K. Lewandowska, and T. Klinkosz (2013). The solution to subdiffusion-reaction equation for the system with one mobile and one static reactant. Acta Phys. Pol. B 44(5), 967-975.

Lewandowska, K. and T. Kosztołowicz (2007). Numerical study of subdiffusion equation. Acta Phys. Pol. B 38, 1847-1854.

Lewandowska, K. and T. Kosztołowicz (2012). Application of diffusion-reaction equations to model carious lesion progress. Physica A 391, 2608-2616.

Maksimovskii, I., I. Nikitina, and A. Kolesnik (1990). A mathematical model of the development of dental caries [in russian]. Stomatologiia 69(2), 5-9.

Metzler, R. and J. Klafter (2000). The random walk's guide to anomalous diffusion: A fractional dynamics approach. Phys. Rep. 339, 1-77.

Shi, Z. and L. Erickson (2001). Model development and simulation of in situ stabilization in lead-contaminated solis. J. Hazard Mater. 87(1-3), 99-116.

Taitelbaum, H., A. Yen, R. Kopelman, S. Havlin, and H. Weiss (1996). Effect of bias on the kinetics of the $a+b \rightarrow c$ with initially separated components. Phys. Rev. E 54(6), 5942-5947.

van Dijk J.W., B. J.M., and F. Driessens (1983). Diffusion in mammalian tooth enamel in relation to the caries process. Arch. Oral Biol. 28(7), 591-597.

Wu, M.-S., W. Higuchi, J. Fox, and M. Friedman (1976). Kinetics and mechanism of hydroxyapatite crystal dissolution in weak acid buffers using the rotatin disk method. J. Dent. Res. 55(3), 496-505.

Zero, D. (1999). Dental caries process. Cariology 43(4), 635-664. 